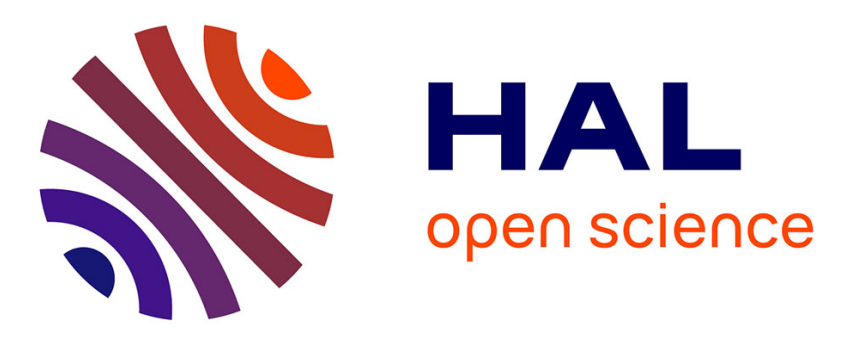

\title{
A Versatile Conducting Interpenetrating Polymer Network for Sensing and Actuation
}

Chia-Ju Peng, Tien Anh Nguyen, Kätlin Rohtlaid, Cedric Plesse, Shih-Jui Chen, Luc Chassagne, Barthélemy Cagneau

\section{- To cite this version:}

Chia-Ju Peng, Tien Anh Nguyen, Kätlin Rohtlaid, Cedric Plesse, Shih-Jui Chen, et al.. A Versatile Conducting Interpenetrating Polymer Network for Sensing and Actuation. IEEE International Conference on Robotics and Automation, May 2017, Singapour, Singapore. hal-01639749

\section{HAL Id: hal-01639749 https://hal.science/hal-01639749}

Submitted on 20 Nov 2017

HAL is a multi-disciplinary open access archive for the deposit and dissemination of scientific research documents, whether they are published or not. The documents may come from teaching and research institutions in France or abroad, or from public or private research centers.
L'archive ouverte pluridisciplinaire HAL, est destinée au dépôt et à la diffusion de documents scientifiques de niveau recherche, publiés ou non, émanant des établissements d'enseignement et de recherche français ou étrangers, des laboratoires publics ou privés. 


\title{
A Versatile Conducting Interpenetrating Polymer Network for Sensing and Actuation
}

\author{
Chia-Ju Peng ${ }^{1,2}$, Tien Anh Nguyen ${ }^{1}$, Kätlin Rohtlaid ${ }^{3}$, Cédric Plesse $^{3}$, Shih-Jui Chen ${ }^{2}$, \\ Luc Chassagne $^{1}$ and Barthélemy Cagneau ${ }^{1}$
}

\begin{abstract}
This work deals with a ConductingInterpenetrating Polymer Network (C-IPN). The C-IPN exhibits very interesting and promising properties which can make it suitable for applications in robotics as a tool to perform tasks in the fields of manipulation, grasping or force measurement. It is known in the literature that such C-IPN may be actuated and bended to interact with other objects. Some of them can also be used as sensors to characterize the interaction. In this paper, we show that actuation and sensing can be performed at the same time. Moreover, we propose analytical models which can be useful for future work to process the C-IPN output and to control them. All results are verified with experimental data.
\end{abstract}

\section{INTRODUCTION}

Gripping and manipulating microscale objects is a critical issue of many applications in micro-assembly [1], microrobotics [2], [3], microfluidics, optics [4] or biology [5], [6]. In micro-manufacturing, assembling the components precisely is essential and is handled with techniques such as MEMS [7], [8]. Several micro-grippers and driving techniques have been developed, including electrothermal actuators [9], [10], electrostatic actuators [11], electromagnetic actuators [6], [12], piezoelectric actuators [13], [14], [15], shape memory alloys (SMAs) [16], and electronic conducting polymers (ECPs) [17], [18], [19]. For high precision and reliability, the requirement of sensorized micro-grippers increases [20], [21]. Grippers with embedded sensors to monitor the gripping force are required to manipulate the objects without any damage. However, the available workspace for additional sensors is limited. Integrating sensors and actuators on the same electrode or using actuators with intrinsic sensing capability can save the volume of the final product. Conducting interpenetrating polymer networks (CIPNs) appear as a potential solution, to design compact grippers that can sense and actuate at the same time with only two wires connected.

C-IPN is a flexible tri-layer structure which comprises two conducting polymer electrodes on each face of the electrolyte membrane. Electronic conducting polymer (ECP) are reactive materials and can be electrochemically oxidized or reduced, while ions exclusion or inclusion from the electrolyte for electroneutrality contributes to volume change

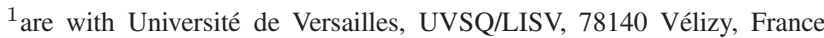
barthelemy . cagneauluvsq. fr

2 are with National central University, Department of Mechanical Engineering, Taoyuan, Taiwan raychen@ec.ncu.edu.tw

3 are with Université de Cergy, LPPI / I-MAT, Cergy-Pontoise, France cedric.plessedu-cergy.fr
}

of the polymer [22], [23]. The related deformation caused by reversible redox reaction makes ECP popular in the family of electroactive polymers (EAPs), attracting wide attention for actuating application [24]. In recent years, researches of ECP actuators using poly(3,4-ethylenedioxythiophene) (PEDOT) [25], [26] or polypyrrole (PPy) [27], [28] have been proposed. Among them, tri-layer devices consisting of two ECP electrodes and an interjacent solid polymer electrolyte (SPE) as an ionic reservoir can operate in open-air [29], [30]. Moreover, C-IPNs with ECP/SPE/ECP layers have been described. This architecture presents several advantages such as cyclability, good performances, fast response and compact size [31].

In this study, we present a C-IPN that may act both as an actuator and as a sensor, having great potential to be a microgripper that actuates and senses the contact at the same time without additional sensor. When electric potential difference is applied between the two electrodes, the ECP electrodes are deformed in opposite direction and bend the conducting C-IPN according to the redox reaction. On the other hand, when the C-IPN is bent by a mechanical stimulus, an electric potential can be measured between the two electrodes due to mechanically induced ion motion between them [32]. The dimensions of C-IPN in this study are in millimeters (for the length and the width) while the thickness is a few hundreds of micrometers. Experimental data are provided but not limited to this scale.

In section II, we briefly present the fabrication process of the C-IPN. More details can be found in [33]. The experimental setup is also presented. In section III, the C-IPN is used as a sensor and experimental results are presented as well as a model. In section IV, a model is proposed for the actuator and we show that contact detection is enabled while actuating. Finally, in section V, a conclusion is drawn and future work is discussed.

\section{C-IPN FABRICATION PROCESS AND EXPERIMENTAL SETUP}

\section{A. Conducting C-IPN Synthesis}

The electroactive material is prepared according to a detailed procedure described elsewhere [33]. The conducting C-IPNs are composed of two ECP electrodes interpenetrated in both faces of a membrane acting as an electrolyte reservoir. The presence of these ions is necessary to make these materials electroactive as actuators and as sensors. 


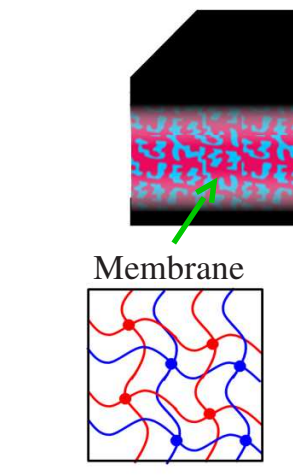

PEO/NBR C-IPN

+ ionic liquid (EMITFSI)
Electroactive Electrodes

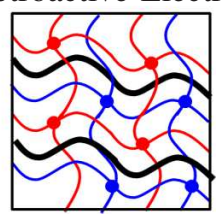

ECP electrode (PEDOT) interpenetrated in $\mathrm{PEO} / \mathrm{NBR} \mathrm{C}-\mathrm{IPN}$
Fig. 1: Schematic representation of the structure and composition of conducting C-IPN used as actuators or/and as sensors

The interpenetrated ECP electrodes, presented in figure 1 are made of poly(3,4-ethylenedioxythiophene) (PEDOT). The membrane is synthesized with a C-IPN architecture with two interpenetrated networks: a poly(ethylene oxide) network (PEO) insuring ionic conductivity through the membrane and a high molecular weight Nitrile Butadiene Rubber (NBR) insuring the mechanical robustness of the final device. After synthesis of the pseudo-trilayer material, the chosen electrolyte, the ionic liquid 1-ethyl-3-methyl-imidazolium bis(trifluoromethylsulfonyl)imide (EMITFSI) is incorporated through a swelling step.

\section{B. Experimental Setup}

The experimental setup is represented in figure 2. It consists of 2 distinct parts. The first one is the C-IPN itself with a rectangular shape $(23 \mathrm{~mm}$ in length, $4 \mathrm{~mm}$ in width and a thickness of $250 \mu \mathrm{m}$ ). It is actuated with an external power supply.

The second part of the experimental setup is a DC motor which can be actuated and controlled to disturb the C-IPN. A rigid probe may be thus in contact with the C-IPN on its two sides.

\section{SENSOR MODE}

In this section, we are focused on the sensing capabilities of the C-IPN. Figure 3 briefly describes the schematic used to interact with the C-IPN. The switch may be placed in position 1 to actuate and to bend the C-IPN and in position 2 for sensing purposes. The terms sensor mode and actuator mode are used hereafter.

\section{A. Electronics Design}

In figure $3, U_{p}$ is the C-IPN voltage and $U_{g}$ is the voltage provided by a signal generator. A resistor $R$ may be tuned to adapt the current consumption as well as the dynamical behaviour of the C-IPN.

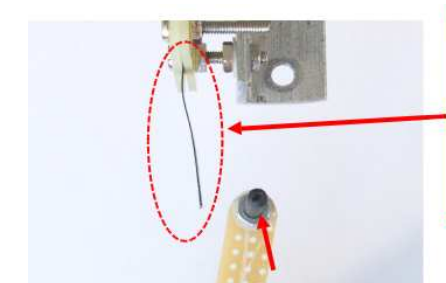

Probe for contact
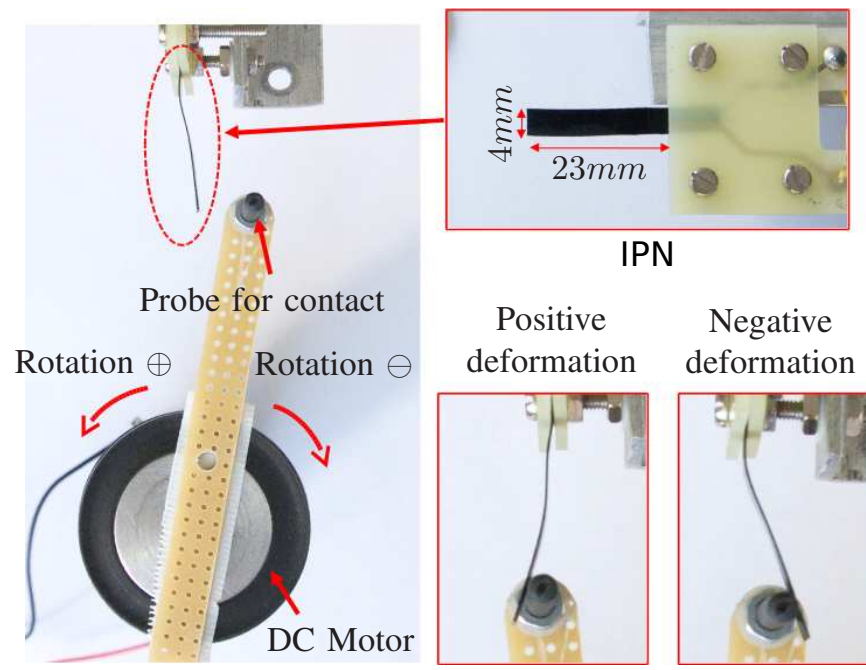

IPN

Positive Negative deformation
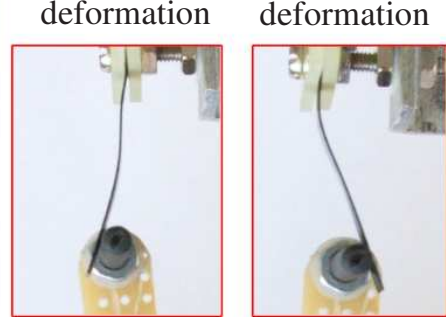

Fig. 2: The C-IPN is actuated with a power supply and disturbances may be applied with a probe connected to a DC motor.

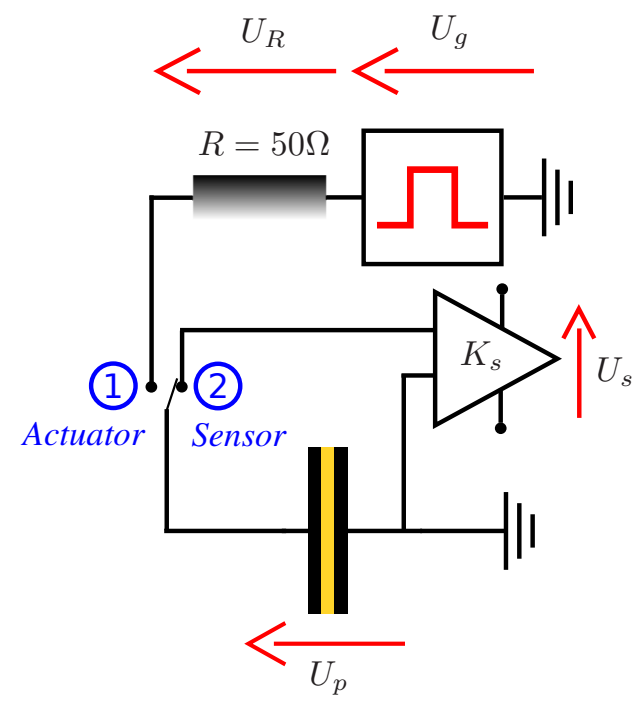

Fig. 3: Illustration of the 2 modes used with the C-IPN.

After first experiments, we can notice that, in sensor mode, $U_{p}$ is only of a few hundreds of microvolts. It is thus necessary to magnify the signal to perform the acquisition. To solve this problem, an analog amplifier circuit with a tunable gain is proposed in figure 4 .

Equation 1 describes the relationship between the input $U_{p}$ and the output $U_{s}$ :

$$
U_{s}(t)=K_{s} U_{p}(t)
$$

The circuit of signal conditioning consists of three successive levels of amplification. To decrease electronic noise, the board uses low-noise operational amplifiers of Analog Device OP27. The board has two levels of non-inverting amplifier tuned with a gain of 11. The intermediate level is a differential amplifier with a gain of 10. It allows the compensation for the offset voltage of the transducer that 


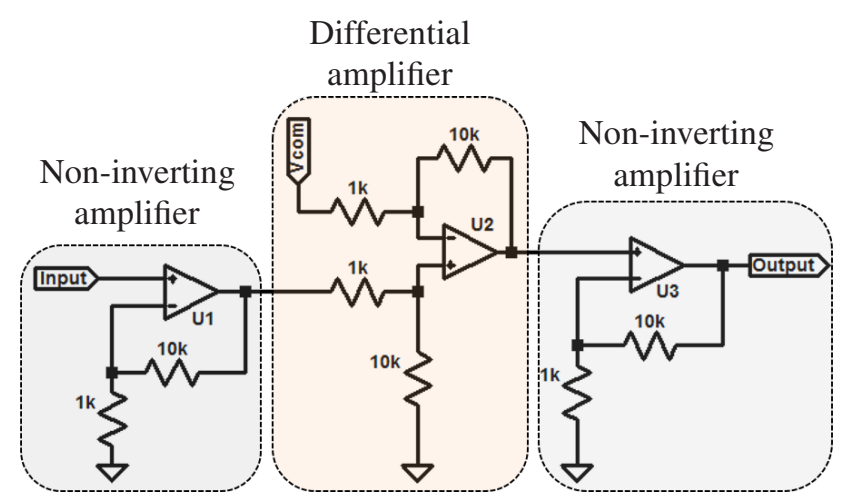

Fig. 4: Electric diagram of the amplifier board. The gain may be tuned and is set to $K_{s}=1210$ for experiments

may occur due, for example, to residual deformations. As a consequence, the signal is amplified with a total gain $K_{s}=1210$.

\section{B. Experimental Results}

We use the DC motor described in section II-B to create passive deformations. The C-IPN is submitted to periodic deformations because of the constant velocity of the motor. After 2 cycles, an opposite voltage is applied to the motor.

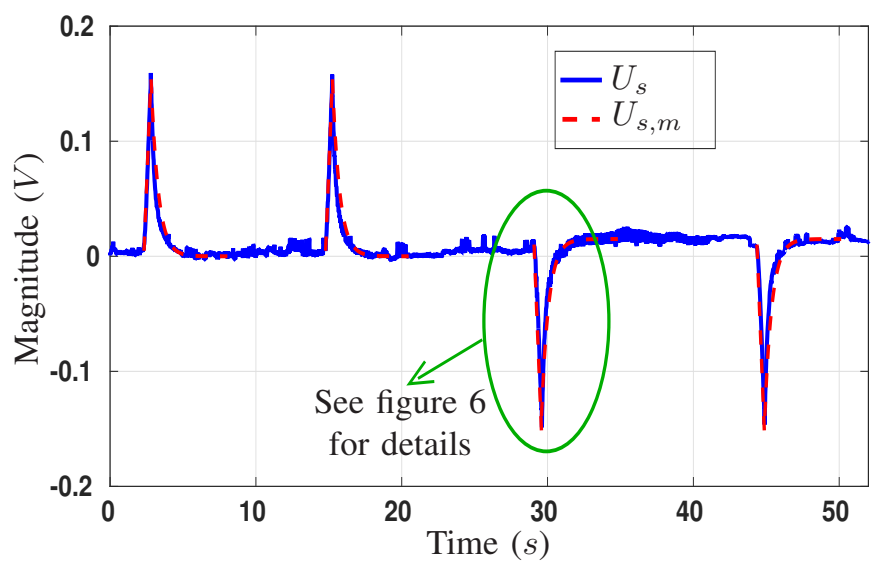

Fig. 5: Amplified sensor output with periodic disturbances. $U_{s}$ is modeled with $U_{s, m}$

Figure 5 clearly presents four cycles which correspond to 4 disturbances applied to the C-IPN. According to equation $1, U_{s}$ is measured and plotted. The amplitude of $U_{s}$ is about $\pm 0.15 \mathrm{~V}$. This sensitivity is high enough to detect the contact while a deformation occurs.

For each disturbance cycle, 2 different phases are observed:

- The C-IPN seems to be first linearly deformed. This phase happens when the probe is in contact with the C-IPN. It is bended until the contact is broken.

- The voltage $U_{s}$ is secondly decreasing with an exponential shape. This phase happens when the C-IPN is relaxing to its initial state.
To validate these observations, we have derived an analytical model $U_{s, m}$ for the two observed phases. The proposed model is such that:

$$
\begin{array}{r}
U_{s, m}(t)=K_{s}\left(t-t_{0}\right), \text { for } 0 \leq t \leq t_{1} \\
U_{s, m}(t)=A_{s} \exp ^{-\left(t-t_{1}\right) / \tau_{s}}+B_{s}, \text { for } t>t_{1}
\end{array}
$$

$P_{s}=\left\{A_{s}, K_{s}, \tau_{s}, B_{s}\right\}$ is a set of parameters that we need to identify with experimental data. $t_{1}$ corresponds to the time when the contact is broken. $t_{0}$ is only a time offset to shift the model with respect to the time $t$. To identify $P_{s}$, we have used the third disturbance cycle in figure 5 .

In figure 6 , the model $U_{s, m}$ is matching experimental data $U_{s}$.

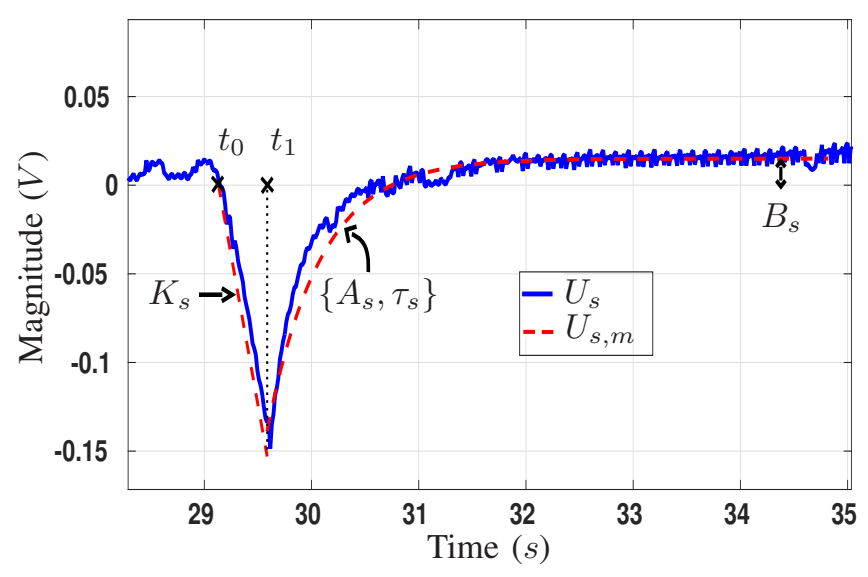

Fig. 6: Zoom on the third cycle

The parameters that we have identified are:

$$
\left\{K_{s}, A_{s}, \tau_{s}, B_{s}\right\}=\left\{-0.32 V . s^{-1}, 0.16 V, 0.5 s, 0.02 V\right\}
$$

It is important to notice that the experiments are reproducible. For the fourth cycle in 5, the set $P$ is the same. When switching to the so-called positive deformations, the signs of $A_{s}$ and $K_{s}$ have been changed to match the sign of $U_{s}$. However, for these first two cycles, $B_{s}=0 \mathrm{~V}$. It seems that the two electrodes (the two sides) of the C-IPN are not symmetric. That would explain the different values of $B_{s}$ depending on the deformations.

To summarize, the amplifier circuit is well suited to detect the deformations that occur on the C-IPN. The electronic board amplifies the C-IPN voltage $U_{p}$ with a gain $K_{s}=$ 1210. However, the amplitudes of negative and positive impulses are not totally identical because of possible asymmetry of electrodes rising from the synthesis. This could explain the asymmetric behaviour of the two electrodes. The error induced by the lack of symmetry is compensated with the $B_{s}$ term in the model (see equation 3 ).

\section{ACTUATOR MODE}

In this section, we are focused on the capabilities of the C-IPN to be used as an actuator. As illustrated in figure 3, the switch is positioned to actuate the C-IPN.

In section III, we have shown that the sensor mode is suitable 
to detect a contact and measure the variations of the forces applied to it. However, the main interest of C-IPN should be to actuate and to sense at the same time.

\section{A. Actuator Modeling}

According to figure $3, U_{g}$ is used to actuate the C-IPN. $U_{g}$ is a square signal with following characterisics:

- Frequency: $f_{g}=0.1 \mathrm{~Hz}$

- Magnitude: $M_{g}= \pm 2 \mathrm{~V}$

At each time, we have also verified that equation 5 is satisfied:

$$
\forall t, U_{g}(t)=U_{R}(t)+U_{p}(t)
$$

In figure $7, U_{g}$ and $U_{p}$ are plotted as functions of time $t$.

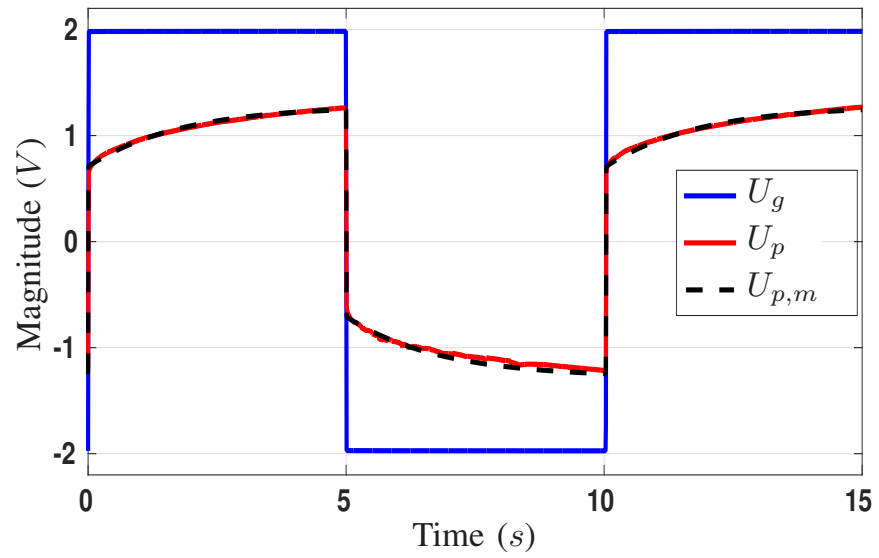

Fig. 7: The C-IPN is actuated with $U_{g} . U_{p}$ is measured and modeled with $U_{p, m}$

While the C-IPN is actuated, a peak value around $200 \mathrm{~mA}$ is observed for the current when $U_{g}$ value is switched. For this experiment, the maximum angle of deformation is $\delta_{\max }= \pm 10^{\circ}$ depending on the sign of $U_{g}$.

One of our concern is to derive a model of the actuator which can be used to understand the C-IPN behaviour. According to figure 7 , it seems that the shape of $U_{p}$ is exponential and we have proposed to derive a model $U_{p, m}$ of $U_{m}$ as:

$$
U_{p, m}= \pm\left(A_{p} * \exp \left(-\left(t-t_{0}\right) / \tau_{p}\right)+B_{p}\right)
$$

The parameters $A_{p}=0.57 \mathrm{~V}, B_{p}=1.28 \mathrm{~V}$ and $\tau_{p}=1.80 \mathrm{~s}$ have been identified. The signs of $U_{p}$ and $U_{p, m}$ only depend on the sign of $U_{g}$.

\section{B. Experimental Results}

In this section, we want to evaluate the capabilities of the C-IPN to actuate and to sense at the same time. The C-IPN is actuated as described in section IV-A with $U_{g}$. We also apply a periodic disturbance on the polymer with the DC motor presented in section II-B. The velocity of the motor is set to a constant velocity so that the frequency $f_{d}$ of the disturbance is $f_{d}=0.5 \mathrm{~Hz}$.

The results are presented in figure 8 .

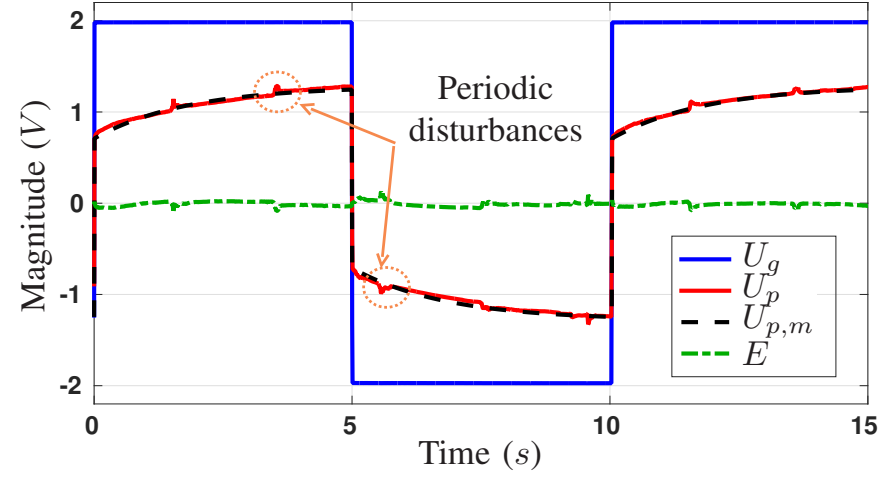

Fig. 8: Periodic disturbances, with a frequency $f_{d}$, are applied to the C-IPN while it is actuated

Results are plotted as functions of time. To emphasize these results, the difference $E$ between the C-IPN voltage $U_{p}$ and its model $U_{p, m}$ is plotted according to equation 7

$$
E(t)=U_{p, m}(t)-U_{p}(t)
$$

$E$ represents the component of $U_{p}$ that corresponds to the disturbance.

The first thing that we can notice is that the model $U_{p, m}$ is still valid for this new experiment. The experiments are indeed reproducible when the same C-IPN is used. The set of parameters needs to be adjusted when the dimensions are changed. The second noticeable thing is that we are able to detect the disturbances applied to the C-IPN while measuring $U_{p}$. All disturbances applied to the C-IPN appear on the plot for 15 seconds. It clearly illustrates that we enable actuation and contact detection at the same time on the same electrodes.

In future work, it should be interesting to quantify the deformations and provide the user with better information concerning the contact between the polymer and its environment. That would make the C-IPN suitable for manipulation. In order to improve the setup, it is however required to adapt the electronic board to amplify the signal component that corresponds to the deformation due to the disturbance.

\section{CONCLUSION AND FUTURE WORK}

In this paper, we have demonstrated the sensing and actuating capabilities of the C-IPN. A remarkable result is that the electronic design and the experimental setup allow to sense and actuate at the same time. However, only binary information like contact/non-contact can be retrieved with this prototype. In future work, we plan to amplify the signal presented in figure 8 to extract precise information about the contact. It will be necessary to process the signal since it contains two components. The first one relates to the contact with a level of a few millivolts. The second one may be of a few volts to actuate the C-IPN.

We are also interested in manipulation at microscales and have developed a C-IPN suitable for this scale. In future work, we hope to demonstrate that similar results can be 
achieved at microscale. One of the main advantage is that the shape of the C-IPN can be freely designed. We can thus expect to use these polymers as micro-grippers to perform manipulation tasks.

\section{ACKNOWLEDGMENT}

This work is made possible with the support of the French National Research Agency (ANR) and the project MicroTIP.

\section{REFERENCES}

[1] J. Agnus, N. Chaillet, C. Clévy, S. Dembélé, M. Gauthier, Y. Haddab, G. J. Laurent, P. Lutz, N. Piat, and M. Rakotondrabe, "Robotic microassembly and micromanipulation at femto-st." Journal of MicroBio Robotics, vol. 8, no. 2, pp. 91-106, apr 2013.

[2] K. Mølhave, T. Wich, A. Kortschack, and P. Bøggild, "Pick-and-place nanomanipulation using microfabricated grippers," Nanotechnology, vol. 17, no. 10, p. 2434, 2006.

[3] M. Gauthier, C. Clévy, D. Hériban, and P. Kallio, "Industrial tools for micromanipulation," in Micro- and Nanomanipulation Tools, Y. Sun and Xinyu, Eds. John Wiley and Sons, 2015, ch. 15, pp. 1-25.

[4] X. Shi, W. Chen, J. Zhang, and W. Chen, "Design, modeling, and simulation of a 2-dof microgripper for grasping and rotating of optical fibers," in 2013 IEEE/ASME International Conference on Advanced Intelligent Mechatronics, July 2013, pp. 1597-1602.

[5] K. Han, S. H. Lee, W. Moon, J. S. Park, and C. W. Moon, "Design and fabrication of the micro-gripper for manipulating the cell," Integrated Ferroelectrics, vol. 89, pp. 77-86, 2007.

[6] T. R. Ger, H. T. Huang, W. Y. Chen, and M. F. Lai, "Magneticallycontrollable zigzag structures as cell microgripper," Lab on a Chip, vol. 13, no. 12, pp. 2364-2369, 2013.

[7] V. Seidemann, S. Butefisch, and S. Buttgenbach, "Fabrication and investigation of in-plane compliant su8 structures for mems and their application to micro valves and micro grippers," Sensors and Actuators a-Physical, vol. 97-8, pp. 457-461, 2002.

[8] J. Cecil, D. Vasquez, and D. Powell, "A review of gripping and manipulation techniques for micro-assembly applications," International Journal of Production Research, vol. 43, no. 4, pp. 819-828, 2005.

[9] M. Rakotondrabe and I. A. Ivan, "Development and force/position control of a new hybrid thermo-piezoelectric microgripper dedicated to micromanipulation tasks," Ieee Transactions on Automation Science and Engineering, vol. 8, no. 4, pp. 824-834, 2011.

[10] K. Carlson, K. N. Andersen, V. Eichhorn, D. H. Petersen, I. Bu, K. Teo, W. Milne, S. Fatikow et al., "A carbon nanofibre scanning probe assembled using an electrothermal microgripper," Nanotechnology, vol. 18 , no. 34, p. 345501, 2007.

[11] O. Millet, P. Bernardoni, S. Regnier, P. Bidaud, E. Tsitsiris, D. Collard, and L. Buchaillot, "Electrostatic actuated micro gripper using an amplification mechanism," Sensors and Actuators a-Physical, vol. 114, no. 2-3, pp. 371-378, 2004.

[12] A. J. Sanchez-Salmeron, R. Lopez-Tarazon, R. Guzman-Diana, and C. Ricolfe-Viala, "Recent development in micro-handling systems for micro-manufacturing," Journal of Materials Processing Technology, vol. 167, no. 2-3, pp. 499-507, 2005.

[13] S. K. Nah and Z. W. Zhong, "A microgripper using piezoelectric actuation for micro-object manipulation," Sensors and Actuators aPhysical, vol. 133, no. 1, pp. 218-224, 2007.

[14] R. Elfrink, T. Kamel, M. Goedbloed, S. Matova, D. Hohlfeld, Y. Van Andel, and R. Van Schaijk, "Vibration energy harvesting with aluminum nitride-based piezoelectric devices," Journal of Micromechanics and Microengineering, vol. 19, no. 9, p. 094005, 2009.

[15] A. M. El-Sayed, A. Abo-Ismail, M. T. El-Melegy, N. A. Hamzaid, and N. A. Abu Osman, "Development of a micro-gripper using piezoelectric bimorphs," Sensors, vol. 13, no. 5, pp. 5826-5840, 2013.
[16] M. Mertmann and E. Hornbogen, "Grippers for the micro assembly containing shape memory actuators and sensors," Journal De Physique Iv, vol. 7, no. C5, pp. 621-626, 1997.

[17] M. Y. Benslimane, H. E. Kiil, and M. J. Tryson, "Dielectric electroactive polymer push actuators: performance and challenges," Polymer International, vol. 59, no. 3, pp. 415-421, 2010.

[18] A. Maziz, C. Plesse, C. Soyer, C. Chevrot, D. Teyssie, E. Cattan, and F. Vidal, "Demonstrating khz frequency actuation for conducting polymer microactuators," Advanced Functional Materials, vol. 24, no. 30, pp. 4851-4859, 2014.

[19] S. Bhattacharya, B. Bepari, and S. Bhaumik, "Ipmc-actuated compliant mechanism-based multifunctional multifinger microgripper," Mechanics Based Design of Structures and Machines, vol. 42, no. 3, pp. 312325, 2014.

[20] J. Park and W. Moon, "A hybrid-type micro-gripper with an integrated force sensor," Microsystem Technologies-Micro-and NanosystemsInformation Storage and Processing Systems, vol. 9, no. 8, pp. 511519, 2003.

[21] D. H. Kim, B. Kim, and H. Kang, "Development of a piezoelectric polymer-based sensorized microgripper for microassembly and micromanipulation," Microsystem Technologies-Micro-and NanosystemsInformation Storage and Processing Systems, vol. 10, no. 4, pp. 275280, 2004.

[22] R. H. Baughman, "Conducting polymer artificial muscles," Synthetic Metals, vol. 78, no. 3, pp. 339-353, 1996.

[23] E. Smela, "Conjugated polymer actuators for biomedical applications," Advanced materials, vol. 15, no. 6, pp. 481-494, 2003.

[24] T. Mirfakhrai, J. D. W. Madden, and R. H. Baughman, "Polymer artificial muscles," Materials Today, vol. 10, no. 4, pp. 30-38, 2007.

[25] A. Khaldi, C. Plesse, C. Soyer, E. Cattan, F. Vidal, C. Legrand, and D. Teyssie, "Conducting interpenetrating polymer network sized to fabricate microactuators," Applied Physics Letters, vol. 98, no. 16, p. 3, 2011.

[26] S. Taccola, F. Greco, B. Mazzolai, V. Mattoli, and E. W. H. Jager, "Thin film free-standing pedot:pss/su8 bilayer microactuators," Journal of Micromechanics and Microengineering, vol. 23, no. 11, 2013.

[27] E. W. Jager, N. Masurkar, N. F. Nworah, B. Gaihre, G. Alici, and G. M. Spinks, "Patterning and electrical interfacing of individually controllable conducting polymer microactuators," Sensors and Actuators B: Chemical, vol. 183, pp. 283-289, 2013.

[28] D. Melling, S. A. Wilson, and E. W. H. Jager, "Controlling the electro-mechanical performance of polypyrrole through 3-and 3,4methyl substituted copolymers," Rsc Advances, vol. 5, no. 102, pp. $84153-84163,2015$.

[29] D. Aradilla, M. M. Perez-Madrigal, F. Estrany, D. Azambuja, J. I. Iribarren, and C. Aleman, "Nanometric ultracapacitors fabricated using multilayer of conducting polymers on self-assembled octanethiol monolayers," Organic Electronics, vol. 14, no. 6, pp. 1483-1495, 2013.

[30] N. Festin, C. Plesse, P. Pirim, C. Chevrot, and F. Vidal, "Electroactive interpenetrating polymer networks actuators and strain sensors: Fabrication, position control and sensing properties," Sensors and Actuators B-Chemical, vol. 193, pp. 82-88, 2014.

[31] C. Plesse, A. Khaldi, Q. Wang, E. Cattan, D. Teyssie, C. Chevrot, and F. Vidal, "Polyethylene oxide-polytetrahydrofurane-pedot conducting interpenetrating polymer networks for high speed actuators," Smart Materials and Structures, vol. 20, no. 12, 2011.

[32] Y. Wu, G. Alici, J. Madden, G. Spinks, and G. Wallace, "Soft mechanical sensors through reverse actuation in polypyrrole," Advanced Functional Materials, vol. 17, no. 16, pp. 3216-3222, 2007.

[33] N. Festin, A. Maziz, C. Plesse, D. Teyssi, C. Chevrot, and F. Vidal, "Robust solid polymer electrolyte for conducting ipn actuators," Smart Materials and Structures, vol. 22, no. 10, p. 104005, 2013. 